\title{
Electrifying surface science
}

Nat. Mater. 17, 592-598 (2018).

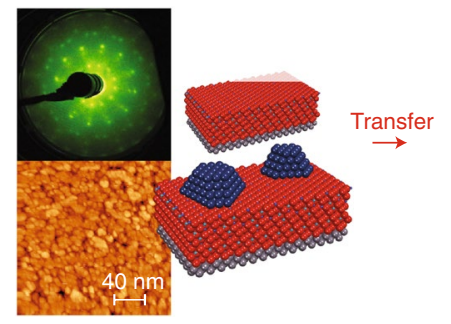

Credit: Springer Nature Limited
Surface science has allowed uncovering many crucial fundamental aspects of heterogeneous catalysis. In combination with model catalysts, it has served as a playground to deepen the atomic-level understanding of single crystals, thin films and supported particles in ultra-high vacuum (UHV) conditions. Surface science studies under electrochemical environments, on the other hand, for their most part have been constrained to single crystal electrodes. Now, Jörg Libuda, Olaf Brummel and co-workers present a general strategy to combine model catalysts prepared in UHV conditions with an electrochemical environment.

The researchers have developed a setup that allows the transfer of the catalyst from the UHV chamber to the electrochemical cell, and back to the UHV chamber, without any contact to ambient atmosphere or foreign materials. Using this clever strategy, the common UHV surface science techniques can be complemented with electrochemical and spectroelectrochemical measurements, and final post analyses can be conducted in the UHV chamber again.
As a proof of concept, they have prepared a model catalyst that consists of platinum nanoparticles supported onto a $\mathrm{Co}_{3} \mathrm{O}_{4}(111)$ film grown on $\operatorname{Ir}(100)$ single crystal by physical vapour deposition in UHV. Low-energy electron diffraction (LEED), scanning tunnelling microscopy (STM) and X-ray photoelectron spectroscopy (XPS) confirm that the atomic structure is preserved in and after the electrochemical environment under a certain range of conditions. Further XPS, infrared reflection absorption spectroscopy (IRRAS) and cyclic voltammetry (CV) studies are carried out to evaluate particle size effects and electronic metal-support interactions under $\mathrm{CO}$ exposure in electrochemical and UHV environments. This exciting strategy will surely trigger many future studies that will bring invaluable contributions to the field.

Marcal Capdevila-Cortada

Published online: 12 July 2018

https://doi.org/10.1038/s41929-018-0122-5 